\title{
Open Journal of Gastroenterology and Hepatology
}

(ISSN:2637-4986)

\section{Polypoid Collagenous Colitis: A Microscopic Colitis with a Macroscopic Appearance}

\section{David Gay, MD1', Darcy Broughel-Baer, DO², Rachel Hudacko, MD}

${ }^{1}$ Department of Pathology, Immunology, and Laboratory Medicine, Rutgers New Jersey Medical School, Medical Science Building C-579, 185 South Orange Avenue, Newark, New Jersey 07103 ${ }^{2}$ Department of Pathology, Orange Regional Medical Center, 707 East Main Street, Middletown, New York 10940

${ }^{3}$ Department of Pathology and Laboratory Medicine, Rutgers Robert Wood Johnson Medical School, MEB 212, 125 Paterson Street, New Brunswick, New Jersey 08901

\section{ABSTRACT}

Collagenous colitis is a type of microscopic colitis which was originally named based on specific histologic features and the lack of macroscopic abnormalities in the colon. However, there are reports in the literature that describe various macroscopic findings on colonoscopy in patients with histologically confirmed

*Correspondence to Author:

Rachel Hudacko, MD

Department of Pathology and Laboratory Medicine, Rutgers Robert microscopic colitis. We report a case of collagenous colitis that Wood Johnson Medical School, was characterized by a diffusely polypoid colonic mucosa on gross examination of a right hemicolectomy specimen that was performed for a benign neoplasm in a 72 year old man. It is important for endoscopists to be aware of the various macroscopic abnormalities that may be present in this "microscopic" disease.

MEB 212, 125 Paterson Street, New Brunswick, New Jersey 08901

How to cite this article:

David Gay,Darcy Broughel-Baer, Rachel Hudacko. Polypoid Collagenous Colitis: A Microscopic Colitis

Keywords: Collagenous colitis (CC), microscopic colitis (MC), colon polyps, endoscopy with a Macroscopic Appearance. Open Journal of Gastroenterology and Hepatology, 2020, 3:36

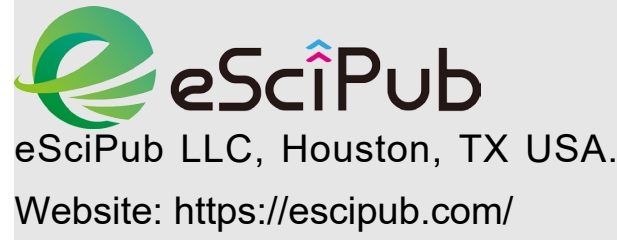




\section{Introduction}

Microscopic colitis (MC) is a cause of chronic, non-bloody, watery diarrhea in older patients, particularly women ${ }^{1}$. As the name suggests, MC is typically not associated with visible macroscopic colonic abnormalities and is diagnosed based on specific histologic findings ${ }^{2}$. The most common symptom is watery diarrhea; however, abdominal pain and weight loss are also common manifestations ${ }^{1}$. MC has been reported to be associated with several autoimmune diseases including rheumatoid arthritis, celiac disease, thyroid disease, and diabetes mellitus ${ }^{1}, 3,4$, as well as certain medications including non-steroidal antiinflammatories, proton pump inhibitors, and selective serotonin reuptake inhibitors ${ }^{1,3,5}$. The pathophysiology of $\mathrm{MC}$ is thought to be an immune reaction to luminal antigens in patients with a possible genetic predisposition, though the cause of this immune dysregulation is still unclear $^{5,6}$.

MC has two subtypes, collagenous colitis (CC) and lymphocytic colitis (LC). CC is defined by the presence of a thickened subepithelial collagen band $\geq 10 \mu \mathrm{m}$, whereas $\mathrm{LC}$ is characterized by an increased number of intraepithelial lymphocytes ( $\geq 20 / 100$ epithelial cells) ${ }^{1,2,7}$. Both subtypes can show increased chronic inflammation in the lamina propria and surface epithelial injury ${ }^{1,2,7}$.

Although the entity is named "microscopic" colitis and was originally believed to show grossly normal colonic mucosa on endoscopy, there have been reports of macroscopic findings with this microscopic disease ${ }^{4,}{ }^{8-13}$. We herein present a case of CC that was characterized by a diffusely polypoid colonic mucosa. There have been only two other cases of $\mathrm{CC}$ presenting with a macroscopic polypoid appearance reported in the literature ${ }^{8-9}$. The differential diagnosis for this finding includes nodular lymphoid hyperplasia of the colon, lymphomatous polyposis, and sessile neoplasms such as sessile serrated polyps and villous adenomas. It is important for endoscopists to be aware of this rare appearance of a not-so-rare disease to ensure appropriate diagnosis and management of the patient.

\section{Case Report}

A 72 year-old man presented to his gastroenterologist for a routine colonoscopy. $\mathrm{He}$ reported diarrhea but denied other changes in bowel habits, rectal bleeding, abdominal pain, or weight loss. His medical history was significant for atrial fibrillation status post ablation, and his medications included aspirin (81 mg daily), atenolol ( $25 \mathrm{mg}$ daily), fish oil (1,000 $\mathrm{mg}$ daily), glucosamine (500 mg daily), and a multi-vitamin. He had undergone a colonoscopy six years prior which demonstrated hemorrhoids and diverticulosis.

On colonoscopy, the patient was found to have a large villous polyp in the cecum that was not amenable to endoscopic resection. The remainder of the colon was reported as unremarkable except for diverticulosis, and he was referred to surgery for a right hemicolectomy. An open right hemicolectomy was performed with resection of an additional segment of transverse colon. On gross examination, there was a distinct $1 \mathrm{~cm}$ polyp in the cecum near the ileocecal valve. In addition, the mucosal surface of the entire right colon showed innumerable sessile, slightly-raised polyps (Fig. 1A). On cross section, the mucosa appeared fibrotic. The submucosa contained edematous fibroadipose tissue, and the muscularis propria and subserosal fibroadipose tissue appeared grossly normal (Fig. 1B). The terminal ileum and appendix were unremarkable. The additional segment of transverse colon showed an unremarkable mucosa and scattered diverticula.

On microscopic examination of the right colon, the mucosa in the depressed areas of the polyps showed an intact surface epithelium with a markedly thickened band of subepithelial collagen, which occupied up to $90 \%$ of the mucosal thickness (Fig. 2A and B). The overall mucosal thickness appeared decreased in these areas. The collagen bands contained entrapped 
capillaries, red blood cells, and chronic inflammatory cells including scattered multinucleated giant cells (Fig. 3). No polarizable foreign material was identified within the giant cells. Chronic inflammatory cells also infiltrated into the surface epithelium. The raised portions of the polyps showed lamina propria edema and surface hyperplastic changes without a significantly thickened collagen band. The depressed areas comprised of atrophic mucosa with thick bands of subepithelial collagen alternating with edematous hyperplastic mucosa imparted an overall polypoid appearance to the mucosal surface (Fig. $4 \mathrm{~A}$ and $\mathrm{B}$ ). These changes involved the entire right colon and one margin of the segment of transverse colon. A Congo red stain confirmed the absence of amyloid deposition. Based on these findings, a diagnosis of polypoid $\mathrm{CC}$ was rendered. Histologically, the cecal polyp was a tubular adenoma with focal high grade dysplasia. Given the diagnosis of $\mathrm{CC}$, the patient was started on budesonide and reported resolution of diarrhea within several weeks.

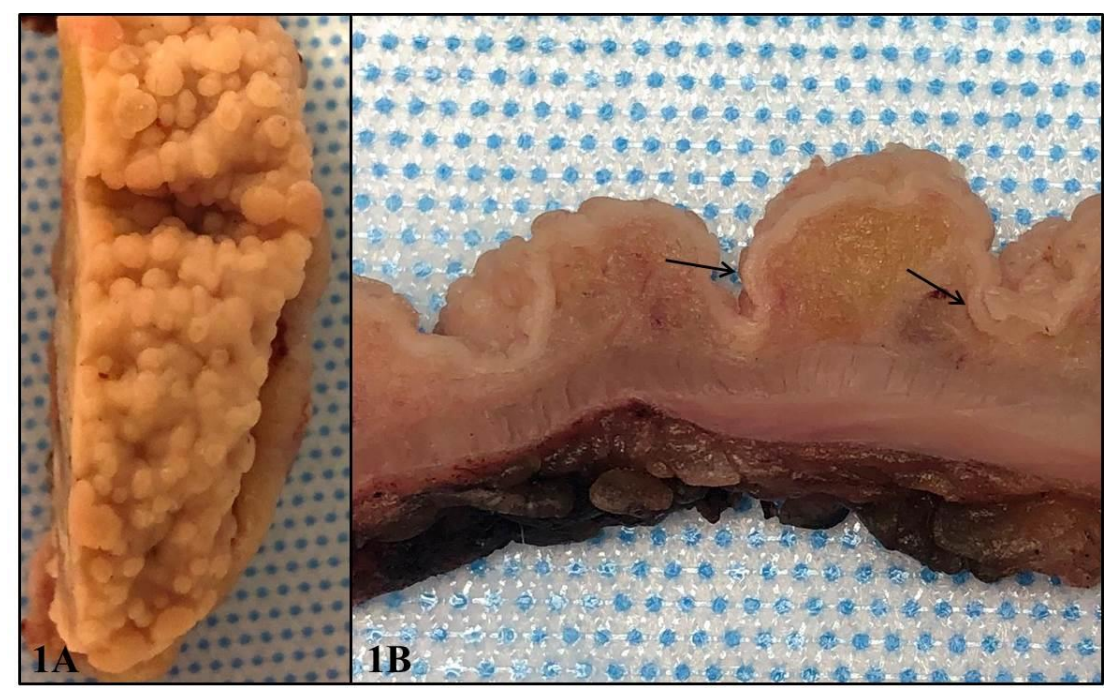

1A: Gross image of the right colon showing a diffusely polypoid mucosal surface. 1B: Cross section of the right colon shows a fibrotic-appearing mucosa (arrows) with submucosal adipose tissue and a normal muscularis proria and subserosa. No true mass lesions are identified.

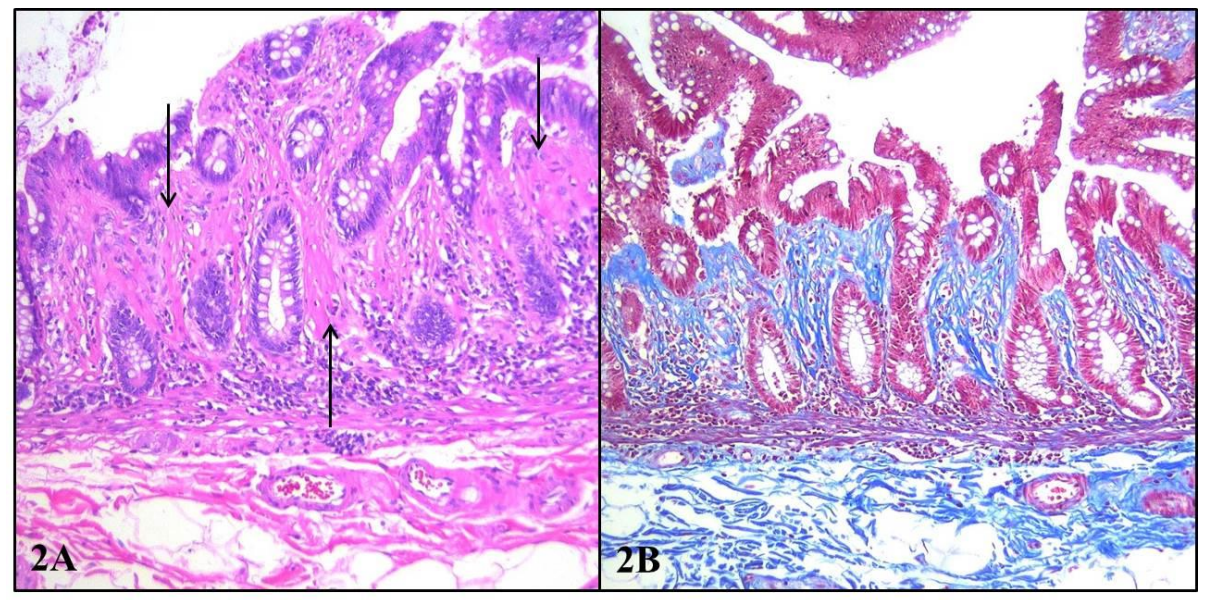

2A: Histologic section through the depressed area of the polypoid mucosa shows a markedly thickened subepithlelial collagen band (arrows) occupying approximately $90 \%$ of the overall mucosal thickness (H\&E stain, 200X). 2B: A trichrome stain highlights the collagen deposition bright blue (200X).

OJGH: https://escipub.com/open-journal-of-gastroenterology-and-hepatology/ 


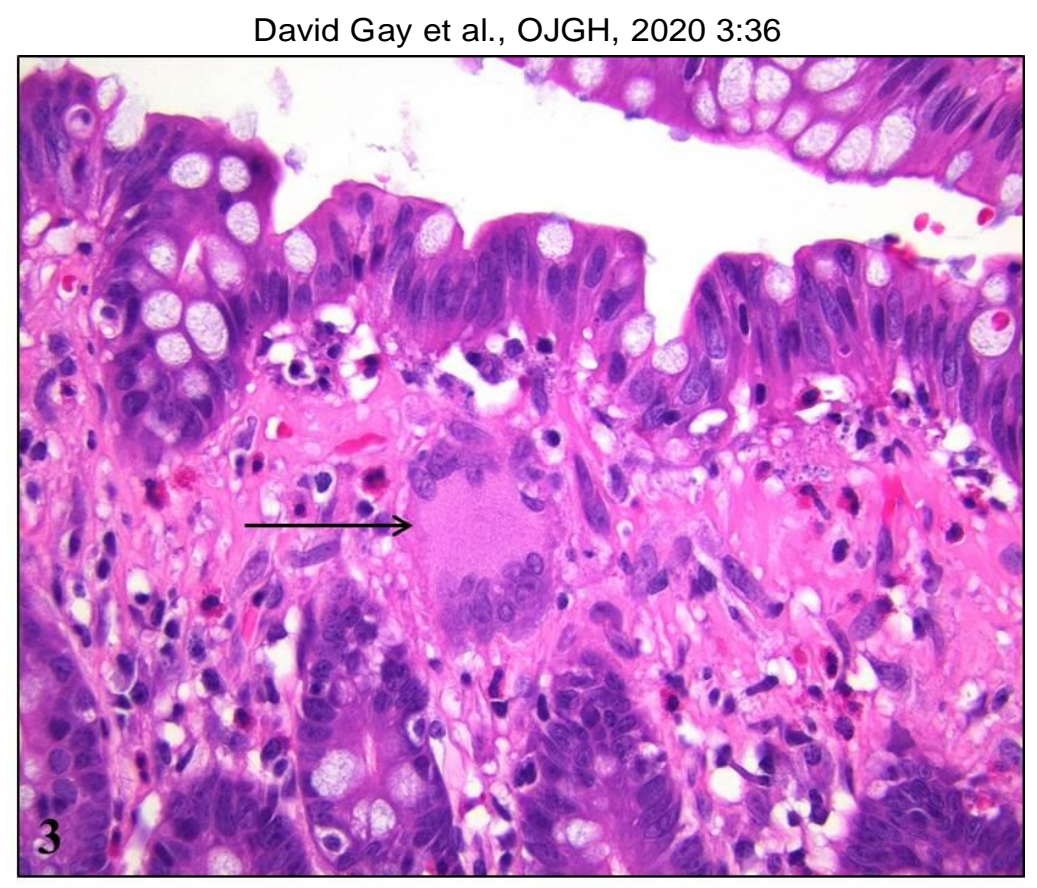

3: Scattered multinucleated giant cells (arrow) were present in the subepithelial collagen band (H\&E stain, 400X).

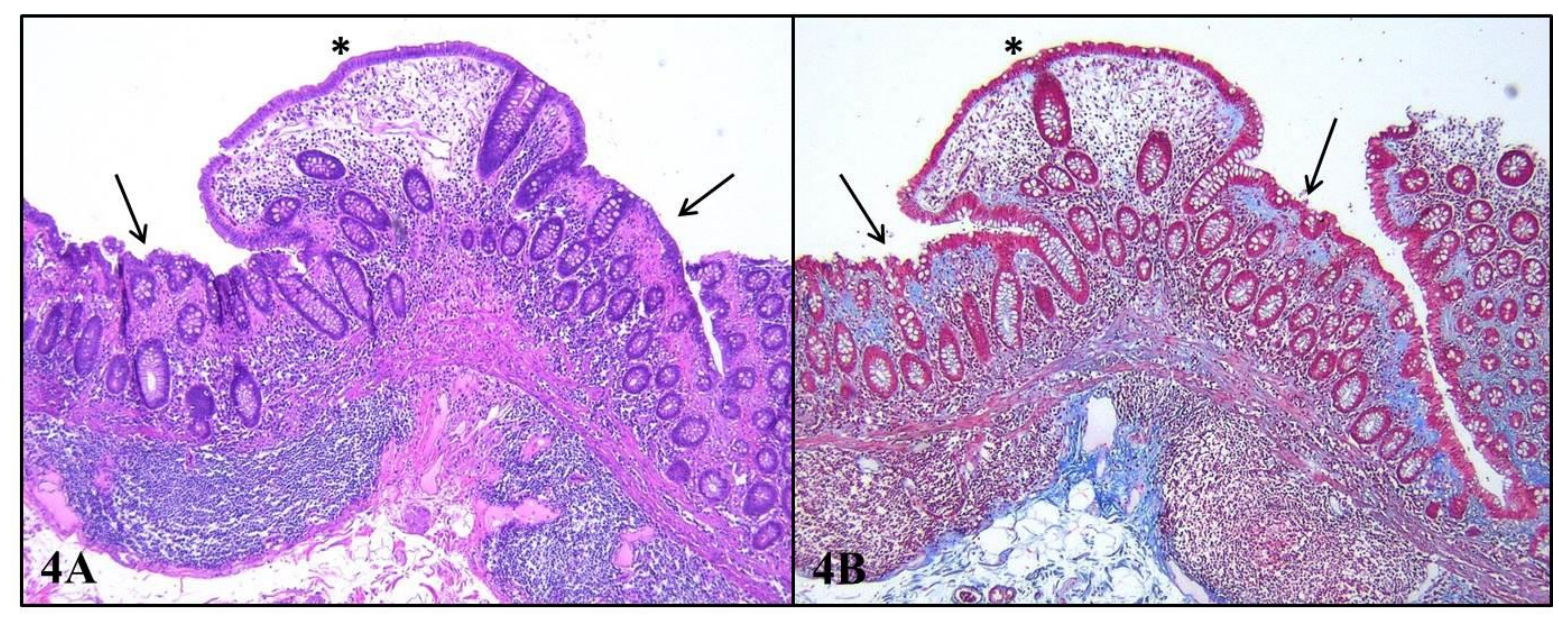

4A: Section through the polypoid mucosa at low power demonstrates thickened subepithelial collagen bands in the depressed areas (arrows) and hyperplastic mucosa with an edematous lamina propria (*) in the raised areas of the "polyps" (H\&E stain, 40X). 4B: A trichrome stain highlights the collagen deposition in the depressed areas bright blue (arrows) and reveals a normal, thin subepithelial collagen layer in the raised areas $\left(^{*}\right)(40 X)$.

\section{Discussion}

The case reported here is of $\mathrm{CC}$ with an unusual polypoid macroscopic appearance of the colonic mucosa. By definition, the gross appearance of CC is typically normal, and the diagnosis is traditionally made on the basis of specific histologic features ${ }^{1,2,7}$. However, there are reports of various macroscopic findings in $\mathrm{CC}$ in the literature, including two case reports with a gross appearance similar to our case ${ }^{8-9}$.

Endoscopically, macroscopic findings in MC may be subtle and detection may depend on the endoscopist's experience. Distinct endoscopic findings, particularly for $\mathrm{CC}$, have been previously described in a systematic review of the literature ${ }^{10}$. Koulaouzidis et al. described four broad categories of endoscopic findings in CC: (i) pseudomembranes, (ii) mucosal vascular 
pattern alterations including an indistinct appearance of blood vessels, crowded tortuous capillaries, and pruning of the mucosal vasculature, (iii) mucosal abnormalities such as red spots, mucosal nodularity, and textural alteration, and (iv) mucosal breaks/tears, including the "cat scratch colon" pattern, longitudinal fractures, fine linear cicatricial lines, and thick scar-like ridges of the mucosal surface $^{10}$. There have also been reports of patients developing mucosal separations and longitudinal tears at the time of colonoscopy due to the pressure of insufflation ${ }^{11-12}$. Colonic perforations have also been described in patients with $\mathrm{CC}$ following colonoscopy and barium enemas ${ }^{13}$.

Notably, there have been only two prior cases reported of $\mathrm{CC}$ with the macroscopic finding of a polypoid colonic mucosa. A report by Smiley et al., described a 53 year-old woman found to have a five centimeter area of nodular mucosa with a "carpet-like appearance", similar to a villous adenoma, near the ileocecal valve on colonoscopy ${ }^{8}$. Freeman et al., reported a 78 year-old woman with a "broad-based, sessile, carpet-like" polypoid lesion at the hepatic flexure on colonscopy ${ }^{9}$. Histologically, this area showed collagenous colitis with subepithelial multinucleated giant cells $s^{9}$. Similarly, our case also demonstrated scattered subepithelial multinucleated giant cells in the collagen band. The presence of giant cells in both CC and LC has been described and is postulated to represent a foreign body giant cell reaction to a luminal agent ${ }^{14}$.

In both of these prior case reports, the polypoid lesions were seen on colonoscopy and were biopsied to reveal a non-neoplastic diagnosis of $\mathrm{CC}$. In our case, the diffusely polypoid mucosa was not noticed on colonoscopy and was subsequently discovered on gross examination of the resection specimen. While the exact reason for the polypoid appearance of $\mathrm{CC}$ in rare cases remains unknown, in our case it seems that the marked thickening of the collagen plate resulted in atrophy of the involved mucosa. This collagen deposition was patchy, and the intervening uninvolved mucosa appeared edematous and hyperplastic, imparting an overall polypoid appearance.

\section{Conclusion}

This case represents an unusual macroscopic presentation of CC, which typically has a normal gross appearance. In our patient, this condition was discovered incidentally after hemicolectomy performed for a tubular adenoma that was not amenable to endoscopic resection. The patient's watery diarrhea was most likely due to the presence of $C C$ and was successfully treated with budesonide after diagnosis. This report adds to the otherwise sparse literature regarding uncommon macroscopic changes that can be seen in CC. Collagenous colitis, although rare, should be a consideration in the differential diagnosis of polypoid lesions, particularly in the clinical setting of older patients presenting with watery diarrhea. By further understanding the variable macroscopic presentation of $\mathrm{CC}$, endoscopists will be better able to identify, diagnose, and treat this condition.

\section{References}

1. Miehlke S, Verhaegh B, Tontini GE, Madisch A, Langner C, Munch A. Microscopic colitis: pathophysiology and clinical management. Lancet Gastroenterol Hepatol 2019; 4: 305-314.

2. Lazenby AJ, Yardley JH, Giardiello FM, Jessurun J, Bayless TM. Lymphocytic ("microscopic") colitis: A comparative histopathologic study with particular reference to collagenous colitis. Hum Pathol 1989; 20(1): 18-28.

3. Loreau J, Duricova D, Gower-Rousseau C, Savoye G, Ganry O, Ben Khadra H, Sarter H, Yzet C, LeMouel JP, Kohut M, Brazier F, Chatelain D, Nguyen-Khac E, Dupas JL, Fumery M. Long-term natural history of microscopic colitis: A populationbased cohort. Clin Trans Gastroenterol 2019;10(9):e00071.

4. Bohr J, Tysk C, Eriksson S, Abrahamsson $\mathrm{H}$, Jarnerot G. Collagenous colitis: a retrospective study of clinical presentation and treatment in 163 patients. Gut 1996;39: 846-851.

5. Beaugerie $L$ and Pardi DS. Review article: druginduced microscopic colitis - proposal for a scoring system and review of the literature. Aliment Pharmacol Ther 2005;22: 277-284.

6. Pardi DS. Diagnosis and management of 
microscopic colitis. Am J Gastroenterol 2017;112: 78-85.

7. Lindstrom CG. Collagenous colitis with watery diarrhea. A new entity? Pathol Eur 1976;11: 8789.

8. Smiley DN and Barkin J. Unusual endoscopic appearance of collagenous colitis. J Clin Gastroenterol 1993;17(1): 84-85.

9. Freeman $\mathrm{HJ}$ and Nimmo M. Carpet-like polypoid lesion in collagenous colitis with mucosal giant cells. Can J Gastroenterol 2011; 25(4): 187-188.

10. Koulaouzidis A and Saeed AA. Distinct colonoscopy findings of microscopic colitis: not so microscopic after all? World J Gastroenterol 2011;17(37): 4157-4165.

11. Kothadia JP, Manatsathit W, and Nakayuenyongsuk W. Spontaneous tear after insufflation: a unique finding of collagenous colitis. Clin Gastroenterol Hepatol 2018;16: e60.

12. Shiratori $Y$ and Fukuda K. Collagenous colitis diagnosed by endoscopically induced mucosal tears. BMJ Case Rep 2019;12: e230570.

13. Marlicz W, Skonieczna-Zydecka K, Yung DE, Loniewski I, Koulaouzidis A. Endoscopic findings and colonic perforation in microscopic colitis: a systematic review. Dig Liver Dis 2017;49(10): 1073-1085.

14. Chang F, Deere $H$, and Vu C. Atypical forms of microscopic colitis: morphological features and review of the literature. Adv Anat Pathol 2005;12: 203-211.

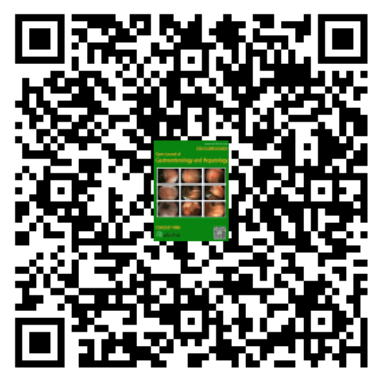

